\title{
PREDICTIVE RE-ALIGNMENT STRATEGY FOR AGILE COMMUNICATION IN WIRELESS SENSOR NETWORKS
}

\author{
Betty Madhurya Vallapuram \\ Radio and Mobile Communication Systems, \\ University of Hertfordshire, \\ Hatfield, UK \\ valpabetty@yahoo.com \\ Gokul P Nair, \\ Radio and Mobile Communication Systems, \\ University of Hertfordshire, \\ Hatfield, UK \\ gokulpnair@outlook.com
}

\begin{abstract}
After the diversification of Wireless Sensor Network (WSN) applications, the need for the most agile communication that can suit the current requirement and scenario is the trend. A number of methodologies to increase the efficiency of communication exist in the literature; however, this is the first attempt to design an agile communication strategy. This paper proposes a predictive re-alignment strategy to facilitate agile communication in the WSNs, where the nodes are capable of small mobility to re-align themselves while communication is on. The standard protocol used to compare the proposed Predictive Re-alignment Strategy (PRS) is Ad-hoc Ondemand Distance Vector Routing (AODV). Simulations show that the PRS has increased the overall network performance by $78 \%$.
\end{abstract}

Keywords: Prediction, Re-alignment, Communication, AODV, WSN

\section{INTRODUCTION}

Wireless Sensor Networks are under research and practical use for over long periods of time for in situ observation [1]. Application areas of Wireless Sensor Networks are many and extending every day from domestic to the most critical purposes. Some of the critical applications include industrial automation and military border monitoring where reliable data delivery is the greatest concern in the communication scenarios. The problem of reliability is a separate field of interest which will not be directly addressed here but will be a by-product of the PRS algorithm proposed.

Energy efficiency is one of the major issues that have been under research interest for a very long time now. But saving energy can also be achieved by small migration of sensor nodes, which is proved to save some energy along with the improvement of network performance. A mobile sink is also introduced to facilitate communication process by initiating node re-alignment process.

Figure 1 shows a simple wireless sensor network that monitors an industrial application scenario. In the industrial area, the sensor nodes are deployed in various regions, and the base station gets reports on the conditions within at regular intervals or at the occurrence of any abnormal incident depending on the application. 


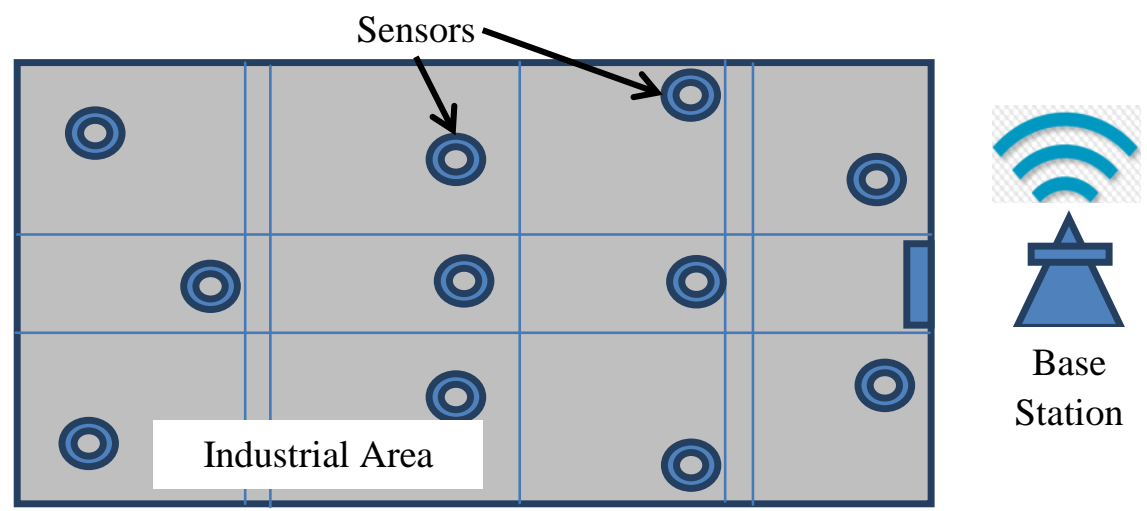

Fig. 1 An Example of WSNs used in an industrial application-overview

\section{RELATED WORK}

Wireless sensor networks have been in use for many years now in different applications. An analysis of the mobility offered by the WSNs describes three main techniques for node mobility according to [2]: Mobile Base Station (MBS), Mobile Data Collector (MDC), and Rendezvous.

- MBS-based solutions: It is a mobile sink capable of changing positions without long-term buffering during operation time. It gets data from the sensors.

- MDC-based solutions: It collects the information over a single-hop wireless transmission. Data's are buffered at source sensor initially. Once the MDC visits the sensors, the download will starts.

- Rendezvous-based solutions: Data is sent dynamically to the rendezvous points which are very near to the mobile devices. It is a hybrid approach.

Data mules were used in [3] to collect data opportunistically. They are collected from sensors within their communication range. Finally, the collected data's are sent to a wireless access point. Due to the random movement of the data mules, the average delay and throughput are not always as good as determined and are likely to vary.

In predictable data collection [4], the data is collected only when the vehicles pass the sensors close by. It was observed that exploitation of data mules helped conserve energy and improve network performance. However, a more reliable strategy will easily replace the current data mules for better performance. A method called, "Energy-Efficient Reliable Routing Considering Residual Energy in Wireless Ad Hoc Networks" was also used for reliable routing [5]. But the reliable communication with mobility in WSNs is still under research, and hence a solution is provided for the same in this paper. The architecture of the sensor networks [6] needs to be better understood before the design of the system.

\section{PROPOSED STRATEGY}

The aim is to produce a self-aligning system that can suit the node positions in a maximum beneficial manner. To bring this into implementation, an algorithm is designed to provide necessary mobility to a node to perform hasslefree communication. In simple words, nodes move around in a controlled manner to get the sensed information reach the base station with great efficiency. Figure 2 
and 3 show the node alignment to obtain maximum efficiency by centring themselves among a group of neighbours

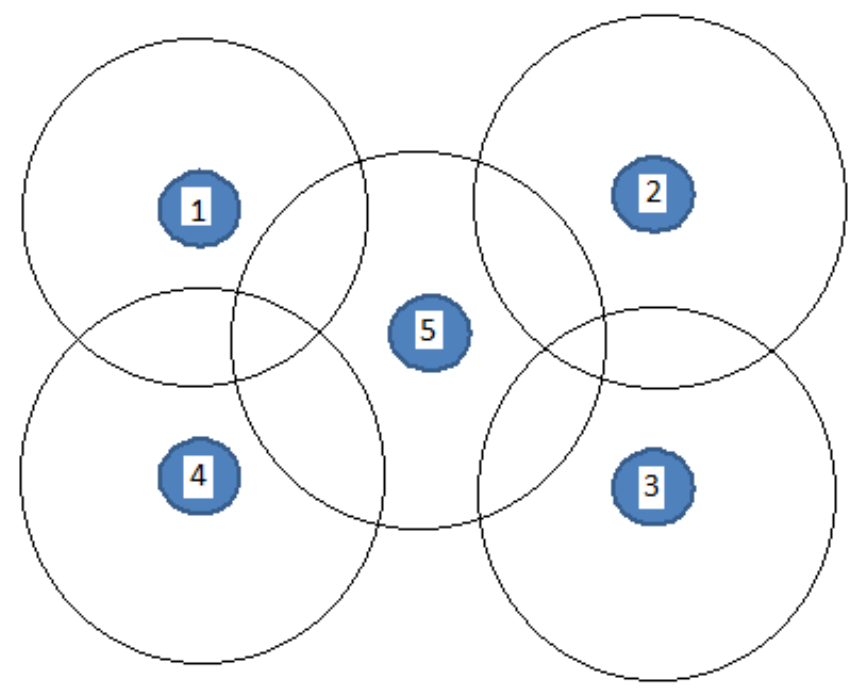

Fig. 2 simple topology of 5 nodes

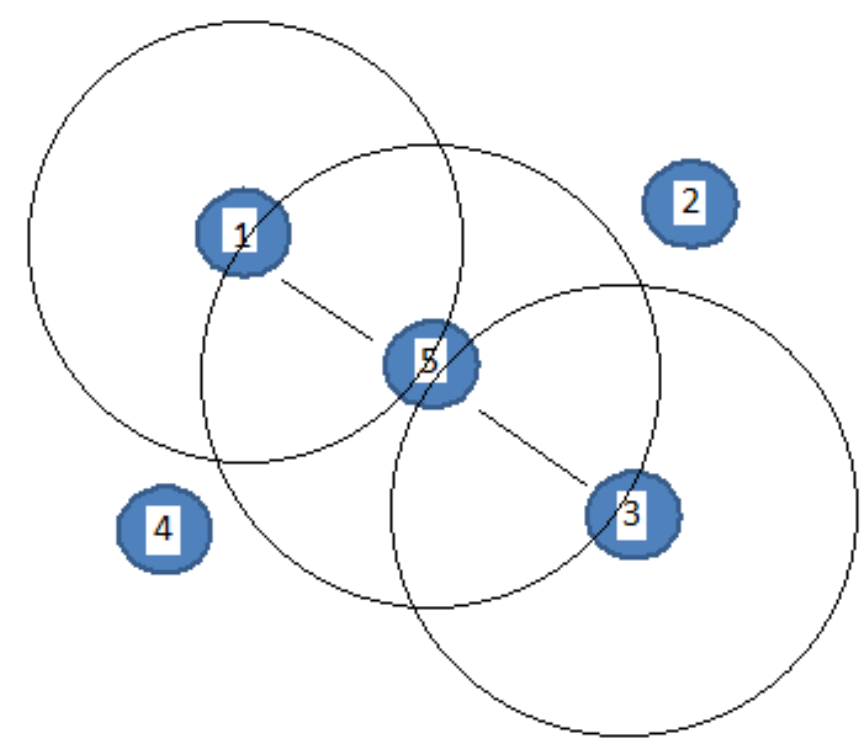

Fig. 3 Nodes 1 and 3 re-aligned to facilitate communication via node 5

Figure 2 shows a simple topology of 5 nodes that do not fall within the range of each other at all at the time of initial node deployment. Communication is not possible here because of transmission range limitation. Figure 3 , on the other hand, shows the possibility of data transfer between nodes 1 and three via node five after the re-alignment of nodes 1 and 3. Similarly, nodes 4 and two can be re-aligned to facilitate communication via node five as shown in Figure 3. 
It is highly impossible to notify the nodes that there is data to be sent across the network when there is no possibility for hearing each other. So in order to fill that gap, we introduce a mobile sink that can transfer request among nodes that cannot hear each other at all and makes a re-alignment decision for the isolated nodes.

The strategy for the communication process using the mobile sink among isolated nodes can be defined by the PRS algorithm.

Step1: Mobile sink on rounds in the node deployment area collects location information of all nodes

Step2: A request to send data by the isolated node is overhead by the mobile sink.

Step3: Mobile sink broadcasts the request after moving closer to the intended destination of the isolated node along with the sender's location and moves on.

Step4: Destination moves towards the direction of sender's location as obtained from the route request.

Step5: For every hop, steps 1 to 4 are repeated until the data reaches the destination, and new locations of nodes are updated in the database of the mobile sink.

\section{SIMULATION ANALYSIS}

Simulation is performed in the network simulator to analyze the efficiency of the PRS algorithm proposed here. Network simulator uses network scenario design in Object-oriented Tool Command Language (OTCL) and C++ for back-end design of the proposed algorithm. Simple AODV protocol is used as a comparison baseline to check the performance improvement. Simulation parameters used for such a simulation process is given in Table 1

TABLE 1 Simulation Parameters of AODV and PRS

\begin{tabular}{|c|c|}
\hline Parameter & Value \\
\hline Simulation Area & $600 \times 500 \mathrm{~m}$ \\
\hline Simulation Time & 20 seconds \\
\hline Channel & Wireless/Phy \\
\hline Radio Propagation Model & Two Ray Ground \\
\hline Number of Nodes & 30 \\
\hline Mobility Model & Random Way Point \\
\hline Protocols Used & AODV and PRS \\
\hline
\end{tabular}

To analyze and compare the efficiency, throughput, packet loss, delay and energy are compared to the PRS and AODV protocols.

\section{A. Throughput}

Throughput is one of the best parameters used to compare the efficiency of a protocol because it gives the actual amount of successful bytes delivered across the network. Figure 4 shows the throughput of the AODV and PRS protocols over 
the simulation time. It can be observed that PRS provides greater throughput over AODV because of the timely re-alignment

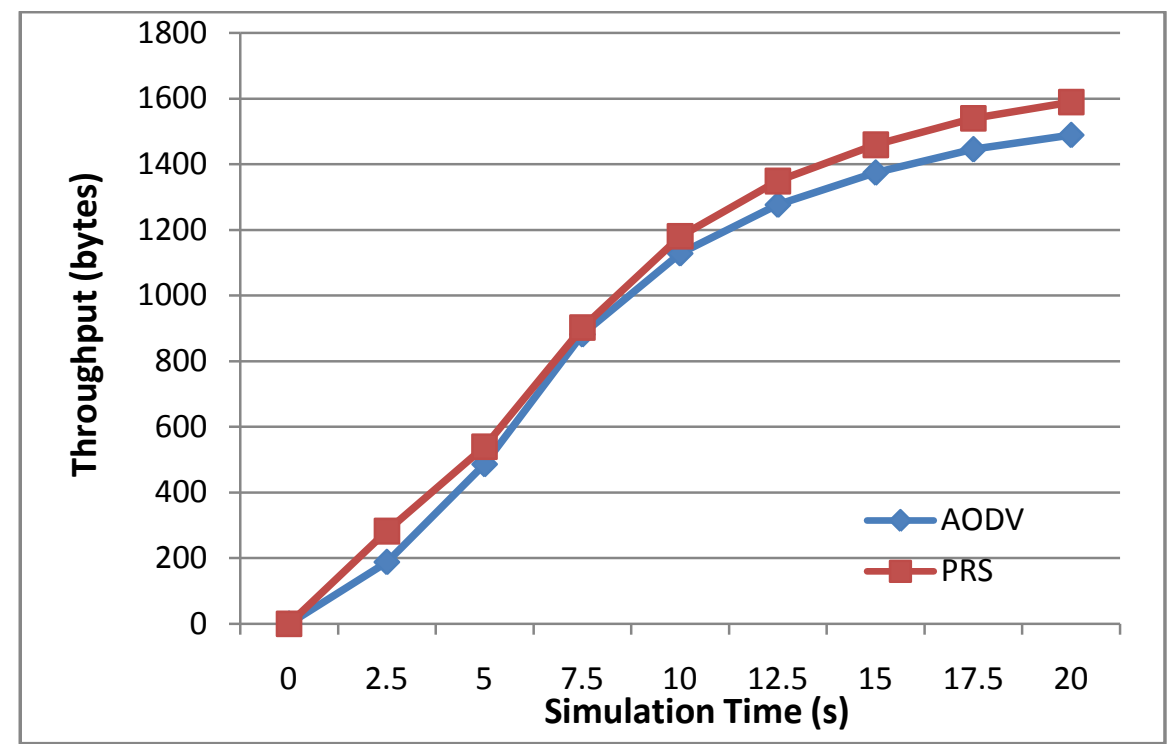

Fig. 4 Throughput of PRS and AODV

\section{B. Packet Loss}

The packets lost during the communication are measured overall in both AODV and PRS protocols, and it was observed that PRS had reduced packet loss by $62 \%$ as shown in Figure 5. In a network of isolated nodes, there would be excessive packet loss when compared to a network with an active mobile sink, which is why there is a visible decrease in the number of packets lost.

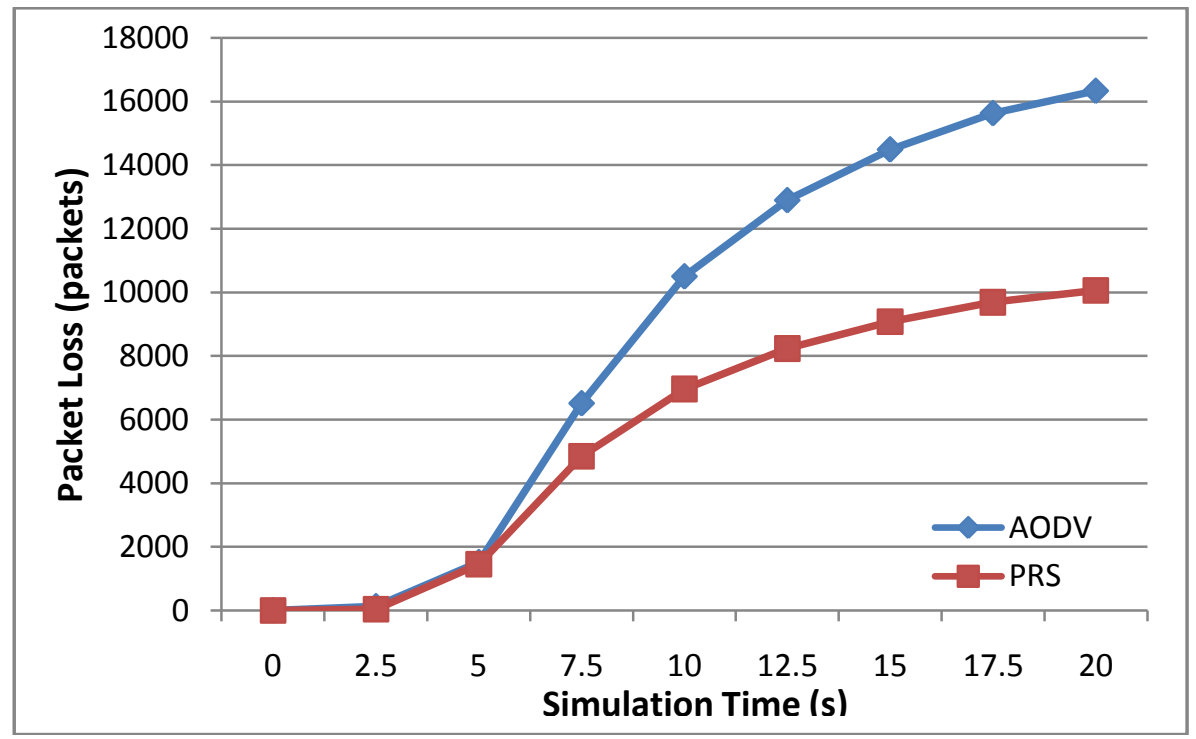

Fig. 5 Packet Loss of PRS and AODV 


\section{Delay}

The overall delay occurred during the transmission of data at every node is the average of the individual delays caused at every node in the network. Hence the delay between the nodes is estimated and plotted in Figure 6 . The average delay occurred during the normal AODV routing is apparently greater than the PRS algorithm proposed.

Clearly, the performance of PRS is greater than AODV due to the realignment of nodes in terms of throughput, packet loss, and delay. This can hence suit many mobile sensor applications that are used for critical monitoring and control purposes

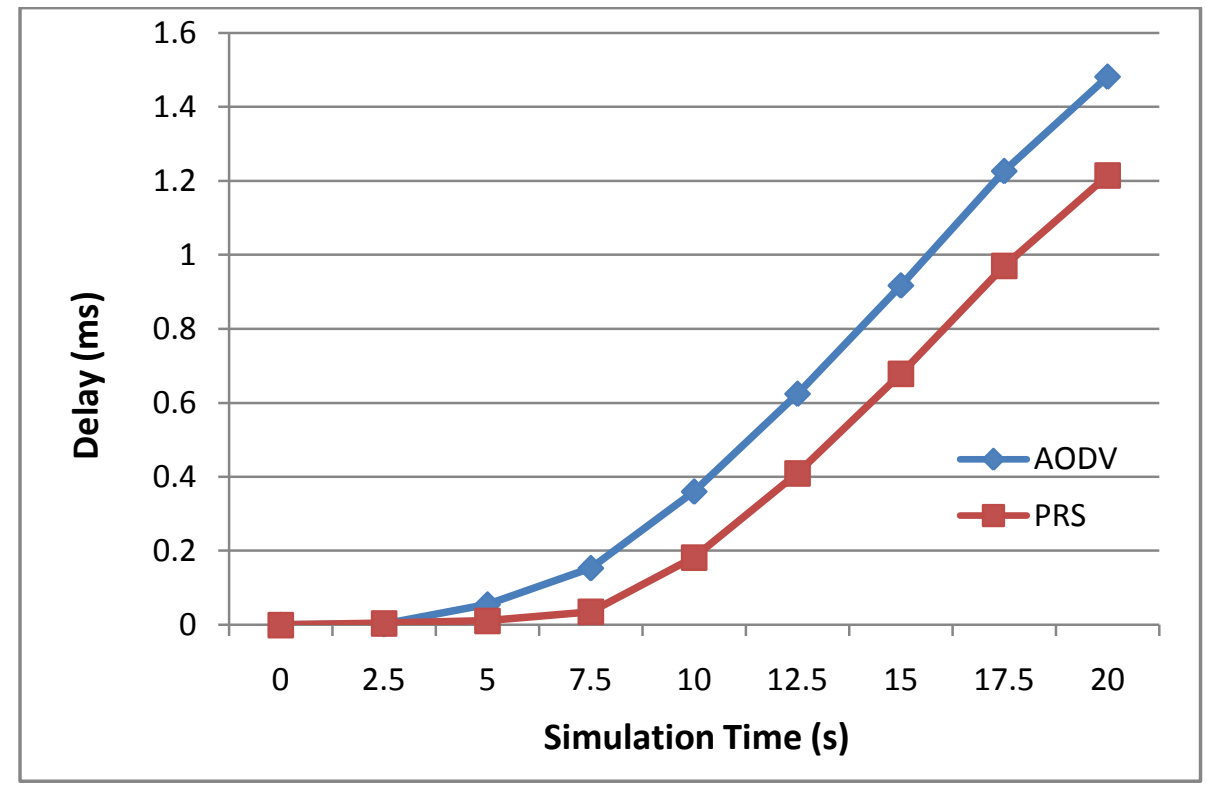

Fig. 6 Delay of PRS and AODV

\section{CONCLUSION}

The PRS for agile communication in WSN was proposed and simulated using the network simulator. From the results obtained through multiple simulations, it can be concluded that the performance of the PRS is much superior to that of the normal AODV. The outputs obtained showed a $78 \%$ difference between the conventional and the proposed scheme. Optimization of the PRS algorithm to compare with some of the recent mobility algorithms is under current research.

Future works will aim at providing a clear-cut application based strategy extended from the PRS with real-time implementation to support the simulation analysis of the same. 


\section{REFERENCES}

[1]. I.F. Akyildiz, W. Su, Y. Sankarasubramaniam, and E. Cayirci, "Wireless sensor networks: a survey", Computer networks, Vol. 38, No. 4, 2002, pp. 393-422.

[2]. E. Ekici, Y. Gu, and D. Bozdag, "Mobility-based communication in wireless sensor networks", IEEE Communications Magazine, Vol. 44, No. 7, 2006, pp. 56-62.

[3]. R.C. Shah, S. Roy, S. Jain, and W. Brunette, "Data mules: Modeling and analysis of a three-tier architecture for sparse sensor networks", Ad Hoc Networks, Vol. 1, No. 2, 2003, pp. 215-233.

[4]. A. Chakrabarti, A. Sabharwal, and B. Aazhang, "Using predictable observer mobility for power efficient design of sensor networks", In Information Processing in Sensor Networks, Springer, Berlin, Heidelberg, 2003, pp. 129-145.

[5]. J. Vazifehdan, R.V. Prasad, and I. Niemegeers, "Energy-efficient reliable routing considering residual energy in wireless ad hoc networks", IEEE Transactions on mobile Computing, Vol. 13, No. 2, 2013, 434-447.

[6]. J. Hill, R. Szewczyk, A. Woo, S. Hollar, D. Culler, and K. Pister, "System architecture directions for networked sensors", SIGPLAN Notices, Vol. 35, No. 11, 2000, pp. 93-104. 